\title{
A Three-Layer Visual Hash Function Using Adler-32
}

\author{
Andysah Putera Utama Siahaan \\ Faculty of Computer Science, Universitas Pembangunan Panca Budi, Jl. Jend. Gatot Subroto Km. 4,5 Sei Sikambing, \\ 20122, Medan, Sumatera Utara, Indonesia \\ andiesiahaan@gmail.com
}

\begin{abstract}
The visual integrity needs to be implemented in sending a picture. There is various image received have no originality. The small change of the pixels does not make the picture content detected by the eye. The integrity validation is very important to be applied. The picture captured by a camera has two dimensions. It is described in pixels such as Width and Length. This study is to validate all the pixels data or the color intensity of both dimensions. If there are a modification in the pixel, this method will give the wrong hash data. The validator will analyze the pixels in every layer such as red, green and blue to ensure the data transmitted is correct. Once there is a slight change in the pixels, the calculation gives the wrong value. It is very useful to compare the image before and after transmission.
\end{abstract}

Keywords: Hash Function, Adler-32, Security.

\section{INTRODUCTION}

Integrity is an aspect that ensures that the data must not be changed without the permission of the authorized competent [3]. For the application of digital imaging, integrity aspect is paramount. It contains the confidential information [2]. Access to data is often sought after by intruders [6][9]. The picture that has been submitted cannot be changed by the unauthorized parties. Violation of this would result in malfunctioning of the validation. It is especially in the fields of education, medicine, military, etc. It needs to prove the originality of the content. Some of them are used as the evidence of a fact. The integrity validation is not only saving someone's life, but it can be implemented on a security side. The highly use of imaging system leads to the data exchange over the air while attaching to the international network. While communication it is imperative to verify the message so that intruder cannot replace with the fake information [5].

For example, when the computer sends the picture, the third party can intercept it in the air, modify the content of the picture and send it to its destination. We should send them with the verification or the message digest.
When the receiver checks the hash of the image, they can compare it to the hash send simultaneously. Sometimes, we do not understand what they are. The information retrieved is used directly without verification. Once we start it, it might run the script consists of some trojan or virus lines. Certain methods of checksums provide the way to verification to ensure the visual integrity [6]. Adler-32 presents to be a practical approach to help manage the originality.

\section{THEORIES}

\subsection{Data Integrity}

Security goals cover three points such as availability, confidentiality, and integrity [7]. A Understanding data integrity broadly refers to the confidence of resource system. Data integrity is paramount because it can ensure the data accuracy, consistency, accessibilities, and the high quality. Following the integrity rules is important. Data with integrity is identical on hold during any operation such as business transfer, storage or retrieval. In simple computer terms, data integrity is the assurance that data is consistent, certified and referenced. Data integrity means that the accuracy and correctness. Data integrity in a database system must be maintained to keep the truth of the stored data. Figure 1 illustrate the scheme of data integrity.

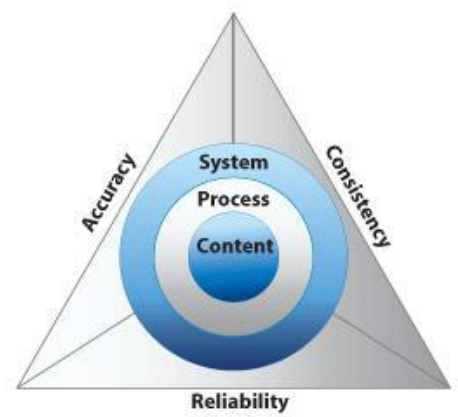

Fig. 1. Data integrity scheme.

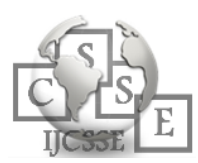


An Example of integrity is the relationship between parents and children. The relationship is noted in a record according to genealogy. If the parent record has one or more of the child records related to all, the database itself will take care of the referential integrity. It automatically guarantees the accuracy, consistensi and reliabily of the data, so there is no record of children raised without parents, and no parent will lose their child's records. It also ensures that no one can remove the parent record while the parents have a record of each child.

\subsection{Hash Function}

A way to test the integrity of the data is to provide a checksum or a sign that data is not changed. The easiest way to do is to calculate the existing characters so that if there is a change, the result will be different. A hash function is a one-way function that produces a "checksum" or "fingerprint" of the data. A message that passes to the hash function will produce output called Message Authenticated Code (MAC). Hash function mapped out a set of data into a limited smaller size. The calculation algorithms uses matrix to map the byte array [1][4]. Let us take a simple example, the mathematical modulus function. The result of the modular expression is the remainder of integer division. For example, "12 mod 5" produces a value of 2 , because 12 divided by 5 to produce a value of 2 and the remainder is 2 . Every day we use modulo operation to express the hours where the modulo is 12 .

The mod operator cannot be used as a good hash function without integrated to the other formula. There are a few requirements to be used practically. For example, the range of the result of the hash function should be enough so that the probability of two different messages will generate the same hash function output. It should be emphasized the word "probability", because there will be two pieces of data that can generate the same hash function output. This is due to the range of hash functions is smaller than the space of the input. To make two messages are intelligible and have the same hash function output is not easy. Another requirement of a good hash function is the change of the character or single bits in the data must produce different output. This property is called avalanche effect.

\subsection{Adler-32}

Mark Adler invented the Adler-32 hash function. He created in 1995 and modified the Fletcher checksum. The length is same as CRC. It offers the speed of validation process. He claimed that Adler-32 is more reliable than Fletcher-16 and slightly less reliable than Fletcher-32. It is obtained by calculating two 16-bit checksums A and B and concatenating their bits into a 32-bit integer. It runs on the hexadecimal platform. A is the sum of all bytes in the stream plus one, and B is the sum of the individual values of $A$ from each step. At the beginning of an Adler-32 run, A is initialized to 1, B to 0 . The sums are done modulo 6552. The bytes are stored in network order, B occupying the two most significant bytes [8].

The function may be expressed as

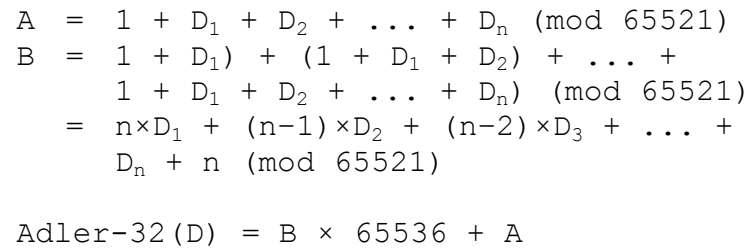

\subsection{Image Preprocessing}

The most important thing in visual hash function is the grayscale process. It is mostly done in the picture processing is changing the color image into the grayscale image, it is used to simplify the model image. The color image consists of three layers, red, green and blue. The grayscale process is to mix the layers and produce a single color layer. When there is a calculation performed using a three-layer, it will be changed by grouping the third layer becomes grayscale and the result is a grayscale image. In this image, there is no color, only the gradation of black and white. There are three ways to get the grayscale intensity.

$$
\begin{aligned}
& \text { Grayscale }=\frac{\max (R, G, B)+\min (R, G, B)}{2} \\
& \text { Grayscale }=\frac{R+G+B}{3}(2) \\
& \text { Grayscale }=(0.21 * R)+(0.72 * G)+(0.07 * B)
\end{aligned}
$$

The formulas above describe how to get the grayscale intensity. Formula 1 concerns to the lightness, Formula 2 concerns to the average and Formula 3 concers to the luminosity. Formula 1 is to find the highest and lowest values of the value of $R, G, B$, then the highest and lowest values are summed and then multiplied by 0.5 . Formula 2 is adding up all the value of $R, G, B$, then divided by 3 , to obtain an average value of $R, G$, and $B$. Formula 3 is to multiply each value of $R, G, B$ with a certain constant predefined value, then the result of multiplying the entire value of $R, G, B$ add up to one another.

\section{PROPOSED WORK}

In this research, we plan to calculate the color intensities of every layer. The color image has three layers of color

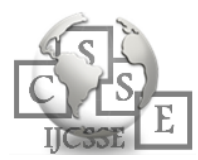


intensities. The red, green and blue layer must be combined and averaged. The average of its color is stored in a grayscale section. We do not build three checksums. However, we combine the three layers into a single converted layer and calculate the pixels. The first step is to split the colors and build the new intensity.

\begin{tabular}{|l|l|l|l|l|}
\hline \multicolumn{5}{|c|}{ RED } \\
\hline $\mathrm{R}^{11}$ & $\mathrm{R}^{12}$ & $\mathrm{R}^{13}$ & $\mathrm{R}^{14}$ & $\mathrm{R}^{15}$ \\
\hline $\mathrm{R}^{21}$ & $\mathrm{R}^{22}$ & $\mathrm{R}^{23}$ & $\mathrm{R}^{24}$ & $\mathrm{R}^{25}$ \\
\hline $\mathrm{R}^{31}$ & $\mathrm{R}^{32}$ & $\mathrm{R}^{33}$ & $\mathrm{R}^{34}$ & $\mathrm{R}^{35}$ \\
\hline $\mathrm{R}^{41}$ & $\mathrm{R}^{42}$ & $\mathrm{R}^{43}$ & $\mathrm{R}^{44}$ & $\mathrm{R}^{45}$ \\
\hline $\mathrm{R}^{51}$ & $\mathrm{R}^{52}$ & $\mathrm{R}^{53}$ & $\mathrm{R}^{54}$ & $\mathrm{R}^{55}$ \\
\hline
\end{tabular}

\begin{tabular}{|l|l|l|l|l|}
\hline \multicolumn{5}{|c|}{ GREEN } \\
\hline $\mathrm{G}^{11}$ & $\mathrm{G}^{12}$ & $\mathrm{G}^{13}$ & $\mathrm{G}^{14}$ & $\mathrm{G}^{15}$ \\
\hline $\mathrm{G}^{21}$ & $\mathrm{G}^{22}$ & $\mathrm{G}^{23}$ & $\mathrm{G}^{24}$ & $\mathrm{G}^{25}$ \\
\hline $\mathrm{G}^{31}$ & $\mathrm{G}^{32}$ & $\mathrm{G}^{33}$ & $\mathrm{G}^{34}$ & $\mathrm{G}^{35}$ \\
\hline $\mathrm{G}^{41}$ & $\mathrm{G}^{42}$ & $\mathrm{G}^{43}$ & $\mathrm{G}^{44}$ & $\mathrm{G}^{45}$ \\
\hline $\mathrm{G}^{51}$ & $\mathrm{G}^{52}$ & $\mathrm{G}^{53}$ & $\mathrm{G}^{54}$ & $\mathrm{G}^{55}$ \\
\hline
\end{tabular}

\begin{tabular}{|c|c|c|c|c|}
\hline \multicolumn{5}{|c|}{ BLUE } \\
\hline $\mathrm{B}^{11}$ & $\mathrm{~B}^{12}$ & $\mathrm{~B}^{13}$ & $\mathrm{~B}^{14}$ & $\mathrm{~B}^{15}$ \\
\hline $\mathrm{B}^{21}$ & $\mathrm{~B}^{22}$ & $\mathrm{~B}^{23}$ & $\mathrm{~B}^{24}$ & $\mathrm{~B}^{25}$ \\
\hline $\mathrm{B}^{31}$ & $\mathrm{~B}^{32}$ & $\mathrm{~B}^{33}$ & $\mathrm{~B}^{34}$ & $\mathrm{~B}^{35}$ \\
\hline$B^{41}$ & $\mathrm{~B}^{42}$ & $\mathrm{~B}^{43}$ & $\mathrm{~B}^{44}$ & $\mathrm{~B}^{45}$ \\
\hline $\mathrm{B}^{51}$ & $\mathrm{~B}^{52}$ & $\mathrm{~B}^{53}$ & $\mathrm{~B}^{54}$ & $\mathrm{~B}^{55}$ \\
\hline
\end{tabular}

Fig. 2. Red, Green and Blue color intensities

The previous figure describes the extracted pixel of a $5 \mathrm{x}$ 5 image length. It splits into three parts. R11 to R55 represents the red color, G11 to G55 represent to the green color while B11 to B55 represent to the blue color. Then the grayscale evaluation converts the values into a single value. We can choose one of the formulas exists. The following equation shows how it performs.

$$
I=\frac{R+G+B}{3}
$$

Where:

$$
\begin{array}{lll}
\text { I } & : & \text { New Intesity } \\
\text { R } & : & \text { Red Color Intensity } \\
\text { G } & : & \text { Green Color Intensity } \\
\text { B } & : & \text { Blue Color Intensity }
\end{array}
$$

\begin{tabular}{|l|l|l|l|l|}
\hline \multicolumn{5}{|c|}{ GRAYSCALE } \\
\hline $\mathrm{I}^{11}$ & $\mathrm{I}^{12}$ & $\mathrm{I}^{13}$ & $\mathrm{I}^{14}$ & $\mathrm{I}^{15}$ \\
\hline $\mathrm{I}^{21}$ & $\mathrm{I}^{22}$ & $\mathrm{I}^{23}$ & $\mathrm{I}^{24}$ & $\mathrm{I}^{25}$ \\
\hline $\mathrm{I}^{31}$ & $\mathrm{I}^{32}$ & $\mathrm{I}^{33}$ & $\mathrm{I}^{34}$ & $\mathrm{I}^{35}$ \\
\hline $\mathrm{I}^{41}$ & $\mathrm{I}^{42}$ & $\mathrm{I}^{43}$ & $\mathrm{I}^{44}$ & $\mathrm{I}^{45}$ \\
\hline $\mathrm{I}^{51}$ & $\mathrm{I}^{52}$ & $\mathrm{I}^{53}$ & $\mathrm{I}^{54}$ & $\mathrm{I}^{55}$ \\
\hline
\end{tabular}

\section{Figure 3. New Intensity}

As we can see in Figure 3, the values inserted into the cells are obtained from the above formula. It aims to reduce the amount of hash function. If we do not combine the color intensities, we have to make the separated hash evaluation and of course, it makes the computer performance slower.

There are three formulas that calculate the Adler-32 Hash to generate the integrity value.

$$
\begin{gathered}
A=1+\sum_{i=0}^{N} I \\
B=\sum_{i=0}^{N} A \\
D=B .65536+A
\end{gathered}
$$

Where:

$$
\begin{array}{ll}
\text { I } & : \text { Grayscale Intensity } \\
\text { A } & : \text { The sum of all bytes } \\
\text { B } & : \text { The sum of the individual values of A } \\
\text { D } & : \text { Adler-32 }
\end{array}
$$

The Adler-32 is obtained by multiplying B to 65536 . The value 65536 is derived from a 16-bit hexadecimal maximum value. It happens since Adler-32 consists of 16-bit (A) and 16-bit (B) sections. The 32 comes from $16+16$

\section{PROPOSED WORK}

This test runs a $15 \times 10$ pixels color image. We try to analyze the Alder-32 hash value if there is a change in color intensity. Figur 4 below shows the original image.

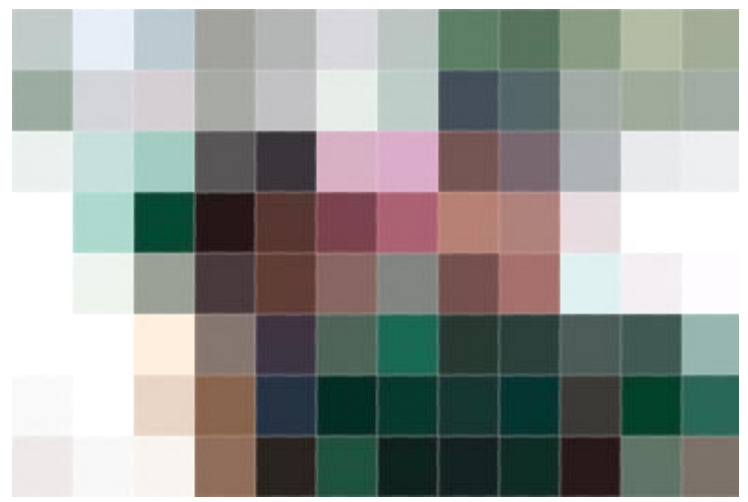

Fig. 4. A $10 \times 15$ image 
Let's take an example, pixel 1 located at cell number 1 $($ Column $=1$, Row $=1)$ consists of $R=192, G=203$ and $\mathrm{B}=202$. The Grayscale is $\frac{192+203+202}{3}=200$. This calculation continues until reach the end of the pixel or reach pixel 150 where the grayscale of the last pixel is $\frac{124+114+105}{3}=114$.

Table 1: Grayscale Intensites

\begin{tabular}{|l|l|l|l|l|l|l|l|l|l|l|l|}
\hline \multicolumn{10}{|c|}{ GRAYSCALE INTENSITIES } \\
\hline 200 & 238 & 199 & 161 & 181 & 216 & 192 & 107 & 99 & 142 & 177 & 161 \\
\hline 162 & 213 & 211 & 168 & 194 & 235 & 197 & 79 & 97 & 166 & 162 & 165 \\
\hline 239 & 214 & 186 & 89 & 57 & 197 & 198 & 96 & 111 & 178 & 234 & 239 \\
\hline 254 & 199 & 40 & 28 & 62 & 90 & 126 & 144 & 144 & 223 & 255 & 254 \\
\hline 255 & 240 & 155 & 64 & 70 & 114 & 131 & 91 & 128 & 235 & 242 & 253 \\
\hline 254 & 254 & 237 & 122 & 60 & 90 & 69 & 48 & 54 & 87 & 77 & 169 \\
\hline 247 & 254 & 214 & 103 & 53 & 28 & 36 & 43 & 35 & 56 & 37 & 76 \\
\hline 235 & 248 & 243 & 115 & 35 & 59 & 26 & 28 & 31 & 29 & 104 & 114 \\
\hline
\end{tabular}

Tabel 1 shows the complete grayscale calculation of the previous image. This table will be the further data to find the Alder-32 value. The value is obtained by applied the earlier Alder-32 formula to these pixels value. Table 2 describes the overall process of Alder-32.

Table 2: Overall process of Alder-32

\begin{tabular}{|r|r|r|}
\hline No. & \multicolumn{1}{|c|}{ A } & \multicolumn{1}{c|}{ B } \\
\hline 1 & 1 & 0 \\
\hline 2 & 201 & 201 \\
\hline 3 & 439 & 640 \\
\hline 4 & 638 & 1278 \\
\hline 5 & 799 & 2077 \\
\hline 6 & 980 & 3057 \\
\hline 7 & 1196 & 4253 \\
\hline 8 & 1388 & 5641 \\
\hline 9 & 1495 & 7136 \\
\hline 10 & 1594 & 8730 \\
\hline 11 & 1736 & 10466 \\
\hline 12 & 1913 & 12379 \\
\hline 13 & 2074 & 14453 \\
\hline 14 & 2236 & 16689 \\
\hline 15 & 2449 & 19138 \\
\hline 16 & 2660 & 21798 \\
\hline 17 & 2828 & 24626 \\
\hline 18 & 3022 & 27648 \\
\hline
\end{tabular}

\begin{tabular}{|c|c|c|}
\hline No. & A & B \\
\hline 38 & 6415 & 122361 \\
\hline 39 & 6614 & 128975 \\
\hline 40 & 6654 & 135629 \\
\hline 41 & 6682 & 142311 \\
\hline 42 & 6744 & 149055 \\
\hline 43 & 6834 & 155889 \\
\hline 44 & 6960 & 162849 \\
\hline 45 & 7104 & 169953 \\
\hline 46 & 7248 & 177201 \\
\hline 47 & 7471 & 184672 \\
\hline 48 & 7726 & 192398 \\
\hline 49 & 7980 & 200378 \\
\hline 50 & 8235 & 208613 \\
\hline 51 & 8475 & 217088 \\
\hline 52 & 8630 & 225718 \\
\hline 53 & 8694 & 234412 \\
\hline 54 & 8764 & 243176 \\
\hline 55 & 8878 & 252054 \\
\hline
\end{tabular}

\begin{tabular}{|r|r|r|}
19 & 3257 & 30905 \\
\hline 20 & 3454 & 34359 \\
\hline 21 & 3533 & 37892 \\
\hline 22 & 3630 & 41522 \\
\hline 23 & 3796 & 45318 \\
\hline 24 & 3958 & 49276 \\
\hline 25 & 4123 & 53399 \\
\hline 26 & 4362 & 57761 \\
\hline 27 & 4576 & 62337 \\
\hline 28 & 4762 & 67099 \\
\hline 29 & 4851 & 71950 \\
\hline 30 & 4908 & 76858 \\
\hline 31 & 5105 & 81963 \\
\hline 32 & 5303 & 87266 \\
\hline 33 & 5399 & 92665 \\
\hline 34 & 5510 & 98175 \\
\hline 35 & 5688 & 103863 \\
\hline 36 & 5922 & 109785 \\
\hline 37 & 6161 & 115946 \\
\hline
\end{tabular}

\begin{tabular}{|r|r|r|}
\hline 56 & 9009 & 261063 \\
\hline 57 & 9100 & 270163 \\
\hline 58 & 9228 & 279391 \\
\hline 59 & 9463 & 288854 \\
\hline 60 & 9705 & 298559 \\
\hline 61 & 9958 & 308517 \\
\hline 62 & 10212 & 318729 \\
\hline 63 & 10466 & 329195 \\
\hline 64 & 10703 & 339898 \\
\hline 65 & 10825 & 350723 \\
\hline 66 & 10885 & 361608 \\
\hline 67 & 10975 & 372583 \\
\hline 68 & 11044 & 383627 \\
\hline 69 & 11092 & 394719 \\
\hline 70 & 11146 & 405865 \\
\hline 71 & 11233 & 417098 \\
\hline 72 & 11310 & 428408 \\
\hline 73 & 11479 & 439887 \\
\hline 74 & 11726 & 451613 \\
\hline
\end{tabular}

\begin{tabular}{|r|c|c|}
\hline No. & A & B \\
\hline 75 & 11980 & 463593 \\
\hline 76 & 12194 & 475787 \\
\hline 77 & 12297 & 488084 \\
\hline 78 & 12350 & 500434 \\
\hline 79 & 12378 & 512812 \\
\hline 80 & 12414 & 525226 \\
\hline 81 & 12457 & 537683 \\
\hline 82 & 12492 & 550175 \\
\hline 83 & 12548 & 562723 \\
\hline 84 & 12585 & 575308 \\
\hline 85 & 12661 & 587969 \\
\hline 86 & 12896 & 600865 \\
\hline
\end{tabular}

\begin{tabular}{|r|c|c|}
\hline No. & A & B \\
\hline 87 & 13144 & 614009 \\
\hline 88 & 13387 & 627396 \\
\hline 89 & 13502 & 640898 \\
\hline 90 & 13537 & 654435 \\
\hline 91 & 13596 & 668031 \\
\hline 92 & 13622 & 681653 \\
\hline 93 & 13650 & 695303 \\
\hline 94 & 13681 & 708984 \\
\hline 95 & 13710 & 722694 \\
\hline 96 & 13814 & 736508 \\
\hline 97 & 13928 & 750436 \\
\hline
\end{tabular}

There are $96(12 \times 8$ pixels $)+1$ calculations. No. $1, \mathrm{~A}=$ 1 and $\mathrm{B}=0$ is the inital state. The last A shows 13928 and the last B shows 750436. The calculation is not ended.

$$
\begin{aligned}
\mathrm{A} & =\mathrm{A} \% \text { MOD_ADLER } \\
& =\mathrm{A} \% 65521 \\
& =13928 \% 65521 \\
& =13928 \\
\mathrm{~B} & =\mathrm{B} \% \text { MOD_ADLER } \\
& =\mathrm{B} \% 65521
\end{aligned}
$$


A. P. U. Siahaan

$$
\begin{aligned}
& =750436 \% 65521 \\
& =29705 \\
\mathrm{AD} & =\mathrm{B} .65536+\mathrm{A} \\
& =29705.65536+13928 \\
& =1946760808(\text { decimal }) \\
& =74093668(\text { hexadecimal })
\end{aligned}
$$

The Adler-32 value showed above is still in decimal format. Alder-32 runs in hexadecimal. The value in hexadecimal is 74093668. It is a combination of two 16bit value. The first section is 7409 and the last is 3668 . When sending this picture to the receiver, the sender must send this Adler-32 value (74093668) simultaneously. Afterward, the recipients synchrony their hash with the sender. Once the value is different, there must be an error or interception while transmitting over the air.

What about if the content has been modified or there are a small undetected object has been inserted into the picture. It is time to prove the hash function. Assume that we modify pixel number 1 . The earlier values are $\mathrm{R}$ $=192, \mathrm{G}=203$ and $\mathrm{B}=202$. The new values are $\mathrm{R}=$ $190, \mathrm{G}=203$, and $\mathrm{B}=201$. It is a small change. It cannot be detected by naked eyes. We just modified the red and blue colors; the green keep similar. The originality can be detected only by using the computer program. That is why the sender always sends the

\begin{tabular}{|c|c|c|}
\hline 17 & 2830 & 24616 \\
\hline 18 & 3024 & 27640 \\
\hline 19 & 3258 & 30898 \\
\hline 20 & 3455 & 34353 \\
\hline 21 & 3534 & 37887 \\
\hline 22 & 3631 & 41518 \\
\hline 23 & 3797 & 45315 \\
\hline 24 & 3959 & 49274 \\
\hline 25 & 4124 & 53398 \\
\hline 26 & 4363 & 57761 \\
\hline 27 & 4577 & 62338 \\
\hline 28 & 4763 & 67101 \\
\hline 29 & 4851 & 71952 \\
\hline 30 & 4907 & 76859 \\
\hline 31 & 5104 & 81963 \\
\hline 32 & 5303 & 87266 \\
\hline 33 & 5399 & 92665 \\
\hline 34 & 5510 & 98175 \\
\hline 35 & 5688 & 103863 \\
\hline 36 & 5922 & 109785 \\
\hline 37 & 6161 & 115946 \\
\hline 38 & 6415 & 122361 \\
\hline 39 & 6613 & 128974 \\
\hline 40 & 6653 & 135627 \\
\hline 41 & 6681 & 142308 \\
\hline 42 & 6743 & 149051 \\
\hline
\end{tabular}
integrity value with the picture; it is to protect the information inside.

Tabel 3: The modified process

\begin{tabular}{|r|r|r|}
\hline No. & \multicolumn{1}{|c|}{ A } & \multicolumn{1}{c|}{ B } \\
\hline 1 & 1 & 0 \\
\hline 2 & 199 & 199 \\
\hline 3 & 438 & 637 \\
\hline 4 & 636 & 1273 \\
\hline 5 & 796 & 2069 \\
\hline 6 & 978 & 3047 \\
\hline 7 & 1195 & 4242 \\
\hline 8 & 1387 & 5629 \\
\hline 9 & 1494 & 7123 \\
\hline 10 & 1593 & 8716 \\
\hline 11 & 1735 & 10451 \\
\hline 12 & 1912 & 12363 \\
\hline 13 & 2073 & 14436 \\
\hline 14 & 2237 & 16673 \\
\hline 15 & 2451 & 19124 \\
\hline 16 & 2662 & 21786 \\
\hline
\end{tabular}

\begin{tabular}{|c|c|c|}
\hline No. & A & B \\
\hline 43 & 6832 & 155883 \\
\hline 44 & 6959 & 162842 \\
\hline 45 & 7103 & 169945 \\
\hline 46 & 7247 & 177192 \\
\hline 47 & 7470 & 184662 \\
\hline 48 & 7725 & 192387 \\
\hline 49 & 7979 & 200366 \\
\hline 50 & 8234 & 208600 \\
\hline 51 & 8474 & 217074 \\
\hline 52 & 8629 & 225703 \\
\hline 53 & 8693 & 234396 \\
\hline 54 & 8763 & 243159 \\
\hline 55 & 8877 & 252036 \\
\hline 56 & 9008 & 261044 \\
\hline 57 & 9099 & 270143 \\
\hline 58 & 9227 & 279370 \\
\hline
\end{tabular}

\begin{tabular}{|r|r|r|}
\hline 59 & 9462 & 288832 \\
\hline 60 & 9704 & 298536 \\
\hline 61 & 9957 & 308493 \\
\hline 62 & 10211 & 318704 \\
\hline 63 & 10465 & 329169 \\
\hline 64 & 10703 & 339872 \\
\hline 65 & 10824 & 350696 \\
\hline 66 & 10884 & 361580 \\
\hline 67 & 10974 & 372554 \\
\hline 68 & 11044 & 383598 \\
\hline 69 & 11092 & 394690 \\
\hline 70 & 11146 & 405836 \\
\hline 71 & 11233 & 417069 \\
\hline 72 & 11310 & 428379 \\
\hline 73 & 11479 & 439858 \\
\hline 74 & 11728 & 451586 \\
\hline 75 & 11982 & 463568 \\
\hline 76 & 12196 & 475764 \\
\hline 77 & 12300 & 488064 \\
\hline 78 & 12353 & 500417 \\
\hline 79 & 12380 & 512797 \\
\hline 80 & 12416 & 525213 \\
\hline 81 & 12459 & 537672 \\
\hline 82 & 12494 & 550166 \\
\hline 83 & 12550 & 562716 \\
\hline 84 & 12587 & 575303 \\
\hline & & \\
\hline 63 &
\end{tabular}

\begin{tabular}{|r|c|c|}
\hline No. & A & B \\
\hline 85 & 12663 & 587966 \\
\hline 86 & 12897 & 600863 \\
\hline 87 & 13145 & 614008 \\
\hline 88 & 13388 & 627396 \\
\hline 89 & 13503 & 640899 \\
\hline 90 & 13538 & 654437 \\
\hline 91 & 13597 & 668034 \\
\hline
\end{tabular}

\begin{tabular}{|r|c|c|}
\hline No. & A & B \\
\hline 92 & 13623 & 681657 \\
\hline 93 & 13652 & 695309 \\
\hline 94 & 13683 & 708992 \\
\hline 95 & 13712 & 722704 \\
\hline 96 & 13816 & 736520 \\
\hline 97 & 13930 & 750450 \\
\hline
\end{tabular}

Table 3 illustrates the process after we modify several color intensities. The modified A shows 13930 and the modified B shows 750450. Moreover, these values are completely different. The calculation is different from the earlier since there was a modification of the byte array.

$$
\begin{aligned}
\mathrm{A} & =\mathrm{A} \% \mathrm{MOD} \_\mathrm{ADLER} \\
& =\mathrm{A} \% 65521
\end{aligned}
$$




$$
\begin{aligned}
& =13930 \% 65521 \\
& =13930 \\
\mathrm{~B} & =\mathrm{B} \% \mathrm{MOD} \text { _ADLER } \\
& =\mathrm{B} \% 65521 \\
& =750450 \% 65521 \\
& =29719 \\
\mathrm{AD} & =\mathrm{B} .65536+\mathrm{A} \\
& =29719.65536+13930 \\
& =1947678314(\text { decimal }) \\
& =7417366 \mathrm{~A} \text { (hexadecimal) }
\end{aligned}
$$

The hexadecimal value is 7417366 A. If we compare to the previous value (74093668) or although we change only 1 bit, the hash value is entirely different. This method is used to testing the level of image originality. Every byte in array is connected each other. If we modify one of them, it affects to the rest.

\section{CONCLUSION}

We wish to thank Mark Adler personally for the Alder32 checksum algorithm. This algorithm runs fast for image processing. The Adler-32 value is obtained by concatenating two 16-bit $\mathrm{A}$ and $\mathrm{B}$. It will be a 32-bit integer. Since it does not use the complex arithmetic expression, it can be applied to the bigger picture. Alder-32 can calculate the originality of what senders send to the recipients. This research does not provide the information hiding; it is only to ensure what the senders send are what the receivers get. The picture can prove anything in real life. So it should be original if used as evidence. His feedback on this research made the algorithm can work together with the image processing.

\section{REFERENCES}

[1] A. P. U. Siahaan, "Three-Pass Protocol Concept in Hill Cipher Encryption Technique," SNATI, Yogyakarta, 2016.

[2] A. P. U. Siahaan, "RC4 Technique in Visual Cryptography RGB Image Encryption,” International Journal of Computer Science and Engineering, vol. 3, no. 7, 2016.

[3] B. Forouzan, Cryptography and Network Security, McGraw-Hill, 2006.

[4] H. Anton dan C. Rorres, Elementary Linear Algebra, 2011: John Wiley \& Sons.

[5] R. Bhanot dan R. Hans, "A Review and Comparative Analysis of Various Encryption Algorithms," International Journal of Security and Its Applications, vol. 9, no. 4, pp. 289-306, 2015.

[6] S. K. Das, G. Sharma dan P. K. Kevat, "Integrity and Authentication using Elliptic Curve cryptography,"

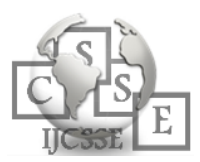

Imperial Journal of Interdiscliplinary Research, vol. 2, no. 5, 2016.

[7] D. Shah, "Digital Security Using Cryptographic Message Digest Algorithm," International Journal of Advance Research in Computer Science and Management Studies, vol. 3, no. 10, pp. 215-219, 2015.

[8] M. Adler, "Wikipedia," Wikipedia, 2232016. [Online]. Available: https://en.wikipedia.org/wiki/Adler-32. [Diakses 87 2016].

[9] A. P. U. Siahaan, "BPCS Steganography Noise-For Region Security Improvisation," International Journal of Science \& Technoledge, vol. 4, no. 6, 2016. 